\title{
Une nouvelle initiative de la SIFEM : des ressources pour s'orienter dans le dédale de la recherche en éducation médicale
}

\author{
A new initiative from the SIFEM : tools for navigating the waters of medical \\ education research
}

En cohérence avec son statut de société savante, l'une des missions premières de la Société internationale francophone d'éducation médicale (SIFEM) est de soutenir le champ de la recherche en éducation médicale et, dans ces mêmes colonnes, nous nous faisions récemment les porte-paroles de son groupe d'action « recherche » ${ }^{[1]}$. Ce groupe s'est réuni lors du dernier congrès de la SIFEM, organisé conjointement en août dernier à Lyon avec la conférence annuelle de l'Association for Medical Education in Europe (AMEE). À cette occasion, ses objectifs ont été reprécisés et de nouvelles orientations stratégiques ont été identifiées ${ }^{[2]}$. L'une d'entre elles vise à rendre plus explicites les finalités de la recherche en éducation médicale, ainsi que les raisons qui justifient de soutenir, de dynamiser et de valoriser ce champ disciplinaire en émergence.

La première raison est liée à l'impact de la recherche en éducation médicale, qui se situe à différents niveaux ${ }^{[1]}$. D'une part, la recherche en éducation médicale permet d'améliorer notre compréhension des phénomènes liés à l'apprentissage et, de ce fait, d'optimiser les pratiques d'enseignement et de soins ${ }^{[1]}$. D'autre part, elle accompagne de manière obligatoire le processus actuel de disciplinarisation de l'éducation médicale, à travers la production d'un savoir propre au champ ${ }^{[1]}$. Enfin, en développant et en diffusant ce savoir, elle participe également à la mise en valeur de la fonction d'enseignement, dans des institutions où la recherche est bien souvent considérée comme prioritaire ${ }^{[3]}$. Par leur nature, ces effets confèrent à la recherche en éducation médicale une forte pertinence et une forte utilité sociales et ils légitiment à eux seuls le soutien apporté par la SIFEM.

Ce soutien se justifie également par le souci d'optimiser les pratiques de recherche en éducation médicale, dont la qualité est encore souvent obérée par une méconnaissance ou par une conception réductrice de ses bases épistémologiques et de ses orientations méthodologiques ${ }^{[1,4-7]}$. En raison de sa filiation historique princeps avec la recherche bioclinique, le paradigme dominant de la recherche en éducation médicale est en effet, en quelque sorte par défaut, le post-positivisme ${ }^{[8]}$. Ainsi, au nom de la conception exclusive de la rigueur scientifique que ce positionnement inspire, toute démarche s'écartant du dogme de l'étude expérimentale, contrôlée - si possible randomisée - est fréquemment considérée comme moins robuste, suscitant immédiatement un procès en suspicion de faible scientificité, au titre de ce que les anglophones appellent la science « molle » $[4,9,10]$. Pourtant, l'essai randomisé contrôlé est rarement applicable dans le cadre de la recherche éducation 
médicale. Les échantillons d'étudiants sont en effet souvent hétérogènes et il n'est pas aisé, ni d'ailleurs toujours pertinent, de justifier la randomisation, en particulier auprès des apprenants eux-mêmes. Par ailleurs, il est souvent illusoire de vouloir isoler l'effet de l'intervention étudiée de celui d'une multitude d'autres facteurs non contrôlables, susceptibles d'influencer les résultats observés, en raison du caractère hautement complexe et contextuellement ancré de l'environnement d'apprentissage ${ }^{[10]}$. L'essai randomisé contrôlé ne permet pas non plus de répondre aux questions importantes dites de «clarification», dont l'objet est de comprendre les multiples facteurs qui interagissent pour déterminer les effets observés $^{[11,12]}$. Il faut avoir à l'esprit que la recherche en éducation médicale ne vise pas seulement à évaluer l'efficacité des interventions pédagogiques; elle tente également de développer des théories permettant de rendre compte de phénomènes complexes tels que l'apprentissage ${ }^{[13]}$. C'est en ce sens que certains chercheurs influents du champ conçoivent la recherche en éducation médicale comme une science sociale et non comme une science biomédicale ${ }^{[14]}$. Au-delà de cette polarisation, les objectifs de la recherche en éducation médicale sont aussi parfois appréhendés comme relevant d'une tension entre un pôle de production de connaissances destinées aux « utilisateurs »- essentiellement les enseignants - et celui d'une production scientifique destinée aux chercheurs eux-mêmes ${ }^{[7]}$.

Dans ce contexte, la publication de la traduction française du guide AMEE ${ }^{\circ} 56^{[15]}$, qui présente une introduction à la recherche en éducation médicale, apparaît comme particulièrement opportune. Ringsted et al. y proposent de baliser le vaste champ de la recherche en éducation médicale en dressant la cartographie de ses différents domaines. Ils utilisent la métaphore de la «boussole de la recherche» pour mettre en exergue l'importance de fixer un cap paradigmatique et méthodologique, permettant de situer la démarche adoptée et d'en cerner les limites. Ils adoptent une posture résolument pragmatique ${ }^{[16]}$, visant à justifier les choix épistémologiques et méthodologiques en fonction de la problématique de recherche. Les auteurs refusent toute hiérarchie entre les différentes méthodologies et discutent des critères de rigueur scientifique appropriés à chaque approche. Ce faisant, ils situent la problématisation scientifique et l'élaboration d'une question de recherche qui en découle comme point de départ à la démarche de recherche, conditionnant les choix méthodologiques. Ainsi, la qualité d'une question de recherche dépendelle fondamentalement de son ancrage théorique, alors même que cette étape est fréquemment négligée par les chercheurs issus du champ biomédical, implicitement convaincus, de façon réductrice, que seule la référence positiviste est légitime pour garantir une «bonne » recherche ${ }^{[1,8]}$.

C'est notamment au regard d'un tel constat que la SIFEM a entrepris de soutenir résolument le champ de la recherche, pour aider les membres de la communauté francophone en éducation des sciences de la santé, et notamment ceux d'entre eux qui ont été formés à la recherche dans le seul paradigme de la recherche bioclinique, à approfondir les bases épistémologiques et méthodologiques qui sont nécessaires pour assurer à la fois la pertinence et la qualité de la recherche en éducation médicale.

La publication de la traduction du guide de Ringsted et al., qui s'inscrit dans le cadre de l'une des actions du partenariat prometteur développé entre la SIFEM et l'AMEE ${ }^{[17]}$, constitue en ce sens une étape fondamentale de la démarche du groupe d'action « recherche ». Elle complète utilement l'offre d'ateliers d'initiation à la recherche en éducation médicale animés par les membres du groupe d'action «recherche », entamée lors du congrès de la SIFEM à Grenoble en 2009, poursuivie lors du congrès de Lyon en 2012 et qui sera reconduite lors du prochain Forum international francophone de pédagogie des sciences de la santé, qui se tiendra à Montréal en mai 2013. D'autres publications, ainsi que des ateliers de perfectionnement, viendront à l'avenir compléter le programme du groupe. Au regard des missions de ce 
dernier, les remarques et suggestions des lecteurs de la revue s'inscrivent de manière évidente comme une contribution nécessaire aux réflexions et aux décisions du groupe.

Valérie DORY ${ }^{1,4}$ et Thierry PELACCIA ${ }^{2,3,4}$

${ }^{1}$ Fonds de la Recherche Scientifique-FNRS, Institut de Recherche Santé et Société, Université catholique de Louvain, Bruxelles, Belgique

${ }^{4}$ Coordonnateurs du groupe d'action « recherche » de la Société internationale francophone d'éducation médicale (SIFEM)

${ }^{2}$ Service d'aide médicale urgente (SAMU 67) et Centre d'enseignement des soins d'urgence (CESU 67) - Hôpitaux universitaires de Strasbourg, Strasbourg, France

${ }^{3}$ Laboratoire de recherche en pédagogie des sciences de la santé, Département de pédagogie médicale Faculté de médecine de Strasbourg, Strasbourg, France

${ }^{4}$ Coordonnateurs du groupe d'action « recherche » de la Société internationale francophone d'éducation médicale (SIFEM)

Mailto : valerie.dory@uclouvain.be Mailto : thierry.pelaccia@wanadoo.fr

\section{Références}

1. Pelaccia T, Dory V, Denef J-F. La recherche en éducation médicale : état des lieux, perspectives et rôle de la Société internationale francophone d'éducation médicale (SIFEM). Pédagogie Médicale 2011; 12:139-48.

2. Denef J-F, Maillard D. Le Congrès AMEE-SIFEM d'août 2012 à Lyon, un moment important dans la vie de notre société. Pédagogie Médicale 2012; 13:221-223.

3. Trigwell K, Shale S. Student learning and the scholarship of university teaching. Stud High Educ 2004;29:523-36.

4. Todres M, Stephenson A, Jones R. Medical education research remains the poor relation. $\mathrm{Br}$ Med $\mathrm{J}$ 2007;335:333-5.
5. Eva KW. Broadening the debate about quality in medical education research. Med Educ 2009; 43:294-6.

6. Norman G. How Bad Is Medical Education Research Anyway? Adv Health Sci Educ 2007;12:1-5.

7. Albert M, Hodges B, Regehr G. La recherche en éducation médicale : entre le service et la science. Pédagogie Médicale 2006;7:73-81.

8. Pelaccia T, Paillé P. La recherche qualitative en pédagogie médicale : histoire, pratique et légitimité. Pédagogie Médicale 2011;12:179-92.

9. Albert M, Laberge S, Hodges BD, Regehr G, Lingard L. Biomedical scientists' perception of the social sciences in health research. Soc Sci Med 2008;66:2520-2531.

10. Gruppen L. Is medical education research "hard" or "soft" research? Adv Health Sci Educ 2008;13:1-2.

11. Cook DA, Bordage G, Schmidt HG. Description, justification and clarification: a framework for classifying the purposes of research in medical education. Med Educ 2008;42:128-33.

12. Norman G. RCT = results confounded and trivial: the perils of grand educational experiments. Med Educ 2003;37:582-4.

13. Regehr G. It's NOT rocket science: rethinking our metaphors for research in health professions education. Med Educ 2010;44:31-9.

14. Monrouxe LV, Rees CE. Picking up the gauntlet: constructing medical education as a social science. Med Educ 2009;43:196-8.

15. Ringsted C, Hodges B, Scherpier A. « La boussole de la recherche »: un introduction à la recherche en éducation médicale. Guide AMEE n56. Pédagogie Médicale 2013;14:49-72.

16. Morgan DL. Paradigms lost and pragmatism regained: Methodological implications of combining qualitative and quantitative methods. J Mix Method Res 2007;1:48-76.

17. Jouquan J. La publication des versions françaises d'une sélection de guides de l'Association for medical education in Europe: un nouveau partenariat entre la SIFEM et l'AMEE. Pédagogie Médicale 2012;13:77-8. 